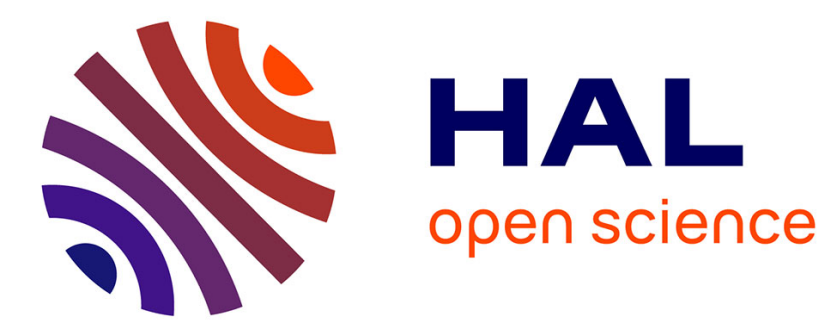

\title{
Sub-grid modelling for a diffusive lattice gas
}

Vincent Labarre, Bérengère Dubrulle, Didier Paillard

\section{To cite this version:}

Vincent Labarre, Bérengère Dubrulle, Didier Paillard. Sub-grid modelling for a diffusive lattice gas. Journal of Physics A: Mathematical and Theoretical, 2020, 53 (40), 10.1088/1751-8121/aba577 . hal02984243

\section{HAL Id: hal-02984243 \\ https://hal.science/hal-02984243}

Submitted on 23 Nov 2020

HAL is a multi-disciplinary open access archive for the deposit and dissemination of scientific research documents, whether they are published or not. The documents may come from teaching and research institutions in France or abroad, or from public or private research centers.
L'archive ouverte pluridisciplinaire HAL, est destinée au dépôt et à la diffusion de documents scientifiques de niveau recherche, publiés ou non, émanant des établissements d'enseignement et de recherche français ou étrangers, des laboratoires publics ou privés. 


\title{
Sub-grid modelling for a diffusive lattice gas
}

\author{
V Labarre ${ }^{1,2}$, B Dubrulle $^{1}$ and D Paillard ${ }^{2}$ \\ ${ }^{1}$ SPEC, CEA, CNRS, Université Paris-Saclay, CEA Saclay, 91191 Gif-sur-Yvette, \\ France \\ ${ }^{2}$ Laboratoire des Sciences du Climat et de l'Environnement, UMR 8212 \\ CEA-CNRS-UVSQ, IPSL and Université Paris-Saclay, 91191 Gif-sur-Yvette, France \\ E-mail: vincent.labarre@lsce.ipsl.fr, berengere.dubrulle@cea.fr
}

\begin{abstract}
We address the sub-grid modeling problem of a simple two-dimensional diffusive lattice gas. We observe that the temporal variation of the coarse-grained current knowing the values of the coarse-grained density, its gradient, and the coarsegrained current is a Gaussian random variable in the low current limit. For this limit, we propose a simple sub-grid model based on a stochastic relaxation equation for the coarse-grained current that takes into account the size of the meshes. The method employed here may be suitable to other systems where we need to represent the smallscale dynamics, as far as the locality of the coarse-grained dynamics is verified.
\end{abstract}

Keywords: Lattice Gas, Statistical Physics, Sub-Grid Modelling

Submitted to: J. Phys. A: Math. Gen. 


\section{Introduction}

Turbulent flows are ubiquitous in natural and industrial flows. Despite their large variety, they all share common characteristics, such as chaotic dynamics (i.e. sensitivity to initial condition) and huge range of scales. The combination of the two properties forbids the prediction of their evolution in a deterministic way, and claims for a statistical description [1]. On the other hand, the smallest scales of the flow are irrelevant for practical purposes, while their resolution represents the most important part of the computational cost. This justifies procedures where only the evolution of the coarsegrained fields is considered, while sub-grid scales are modelled. There is still no universal approach to represent the effect of unresolved scales on the resolved ones. The problem couples the dynamics and the coarse-graining procedure. All methods need, at some point, to introduce empirical parameters (eddy viscosity models [2]) and/or idealized physical hypotheses (see for example [3]). This may lead to unwanted drawbacks, like non-conservation of energy or uncontrolled dependence of the empirical parameters with the coarse graining (in the Smagorinski model for example [4], the size of the mesh in the numerical grid is introduced for dimensional consistency).

Arbitrariness of the modelling could be decreased using systematic and well controlled procedure, that includes coarse-graining. One major difficulty relies on the nonequilibrium nature of the turbulence, in which all physical quantities (mass, momentum, energy, tracers) flow into a system due to an external forcing. While there is presently no systematic out-of-equilibrium theory for turbulence, there has been huge progress in characterization of out-of-equilibrium properties of simplified statistical models. Those models fall into two categories. First, Lattice Gas Cellular Automata (LGCA) are a discrete space-time models version of kinetic theory of gas $[5,6,7,8]$. It was shown, by using classical tools of kinetic theory, that they are able to represent real flow under reasonable approximations. Second, Stochastic Lattice Gases (SLG) have been introduced in several contexts (DNA modelling, traffic flows, paradigmatic non-equilibrium systems) [9]. They have the particularity to be conceptually simple enough to allow detailed computations and complicated enough to represent some interesting features like phase transitions. For example, the detailed statistics of density and current has been derived for some one-dimensional models using the Matrix Anzatz [10], or the Bethe Anzatz [11]. It was also shown that we can compute the probability of observing atypical density and current profiles directly at the macroscopic level (i.e. computing the large deviation functional of the density and current) in the stationary state using the Macroscopic Fluctuation Theory $[12,13,14,15]$. Both LGCA and SLG represent ideal bench test for conceptual ideas on sub-grid modelling because they are conceptually far more simple than a real fluid and are easily simulated. The main difference between LGCA and SLG is that LGCA are not solvable analytically (except in the lattice Boltzmann approximation in some case [16]). 
The goal of the present study is to look for an improved understanding of basic subgrid modelling, by using a simplified model of diffusion based upon a two-dimensional diffusive LGCA. In classical turbulence theory, such systems would be naturally modelled using an eddy viscosity. Here, we show that the model is actually more involved, while still conceptually simple. Our procedure relies upon numerical analysis of the typical behaviour of the coarse-grained current in a two-dimensional diffusive LGCA as a function of the coarse-grained density, its gradient, and the size of the cubic spatiotemporal coarse-graining window. We show that the temporal variation of the coarsegrained current, at fixed coarse-grained density and its gradient, is a Gaussian random variable whose average is given by a relaxation to the hydrodynamic current at a given rate and root mean square that are strongly influenced by the coarse-graining. Using these observations, we propose a simple sub-grid model to describe the evolution of the coarse-grained current.

The outline of the paper is as follows. In a first part, we present our model (part 2). In a second part, we present our numerical results for the conditional PDF of the temporal variation of the coarse-grained current (part 3) and we propose a simple stochastic sub-grid model for the coarse-grained current. We discuss our results and perspectives in part 4. The derivation of the evolution equation of the average density is given in the Appendix A.

\section{Model}

\subsection{Microscopic dynamics}

We study a variant of the model introduced by Hardy, de Pazzis and Pomeau [17, 18] (HPP model). It consists on a square lattice $\mathcal{L}$ of $L \times L$ nodes (see figure 1). Each node consists in four channels which correspond to the velocities of the model $\left(c_{1}=(1,0), c_{2}=(0,1), c_{3}=(-1,0), c_{4}=(0,-1)\right)$. Occupation of the channel $i$ at discrete time $t_{*}$ and discrete position $r_{*}=\left(x_{*}, y_{*}\right)$ is noted $n_{i}\left(r_{*}, t_{*}\right)$. A channel can be occupied by one particle of unit mass or can be empty (exclusion principle), such that $n\left(t_{*}\right)=\left(n_{i}\left(r_{*}, t_{*}\right), i=1, \ldots, 4, r_{*} \in \mathcal{L}\right)$ is the Boolean field that gives the configuration of the system at time $t_{*}$. At each discrete time step, particles at a same node are collided and propagated to nearest nodes according to their velocities. Collision changes a precollision state $s=\left(s_{i}, i=1, \ldots, 4\right)$ to a post-collision state $s^{\prime}=\left(s_{i}^{\prime}, i=1, \ldots, 4\right)$ with

probability $A_{s^{\prime} s}$ where $s_{i}$ is the occupation of channel $i$ (Boolean variable). Here, we consider two types of collisions. Frontal collision occurs with a probability $p$ when there is two particles with opposite velocities at the node. This collision conserves mass and momentum. Three particles collision occurs with a probability $q$. It consists to flip the velocity of the particle which does not face another one. This collision conserves mass but not momentum. It induces a tendency of particles to turn round more frequently when going in high density regions (where three particles collisions occurs more frequently). 
(a)

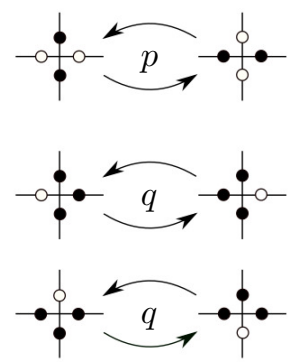

(b)

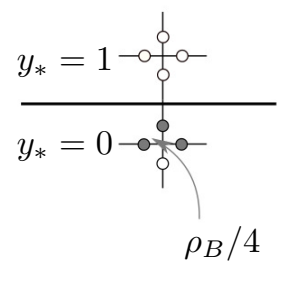

$(c)$

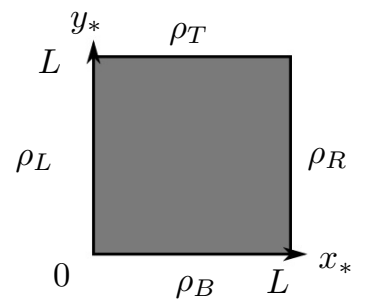

Figure 1. (a) Collision rules of the model. The two particles collision occurs with a probability $p$, and three particles collision occurs with a probability $q$. (b) Bottom boundary condition: at $y_{*}=0$, channels 1,2 and 3 are filled randomly with probability $\rho_{B} / 4$ and particle in cell 4 come from the bulk so $\rho_{B}$ represents the density of the bottom reservoir. Similar conditions are applied on the other boundaries with different densities for the reservoirs. (c) View of the lattice of $L \times L$ nodes between reservoirs at densities $\rho_{L}\left(x_{*}=0\right), \rho_{B}\left(y_{*}=0\right), \rho_{R}\left(x_{*}=L\right)$ and $\rho_{T}\left(y_{*}=L\right)$.

This collision destroys spurious invariants like the particles-holes symmetry (duality) and obviously the conservation of horizontal (vertical) current along horizontal (vertical) lines. The three particle collision is the main difference between our model and the HPP model. These collision rules respect the detailed balance $\left(A_{s^{\prime} s}=A_{s s^{\prime}} \forall s, s^{\prime}\right)$, and hence semi-detailed balance:

$$
\sum_{s^{\prime}} A_{s s^{\prime}}=\sum_{s^{\prime}} A_{s^{\prime} s}=1, \quad \forall s,
$$

Since only the number of particles is conserved by collisions, we observe a diffusive behaviour at large scales. Propagation consists to move particles to neighbouring nodes according to their velocity $n_{i}\left(r_{*}\right) \rightarrow n_{i}\left(r_{*}+c_{i}\right)$. If $p=q=1$ then the evolution of the system inside the bulk is deterministic. At the boundaries, particles are injected in vacant channels (i.e. those that are not filled by particles propagated from the bulk) with a probability equal to the density of the reservoir divided by four (the number of channels per node). We introduce the microscopic observables

$$
\begin{aligned}
& \rho_{*}\left(r_{*}, t_{*}\right)=\sum_{i=1}^{4} n_{i}\left(r_{*}, t_{*}\right) \quad=0,1,2,3,4, \\
& j_{* \alpha}\left(r_{*}, t_{*}\right)=\sum_{i=1}^{4} n_{i}\left(r_{*}, t_{*}\right) c_{i \alpha} \quad=-1,0,1,
\end{aligned}
$$

which are the microscopic density and the microscopic current of particles in the $\alpha$ 's direction.

To obtain the macroscopic evolution of Lattice gas $[5,7,8]$, we introduce an ensemble average procedure $\langle\cdot\rangle$ that connects the discrete, microscopic state with the 
macrostate which is assumed to vary slowly (at least in the limit of large lattice size and large time). We introduce

$$
N_{i}=\left\langle n_{i}\right\rangle, \quad \rho=\left\langle\rho_{*}\right\rangle, \quad j_{\alpha}=\left\langle j_{* \alpha}\right\rangle,
$$

that are the average population of particle with speed $c_{i}$, the average density, and the $\alpha$ 's component of the average current of particles.
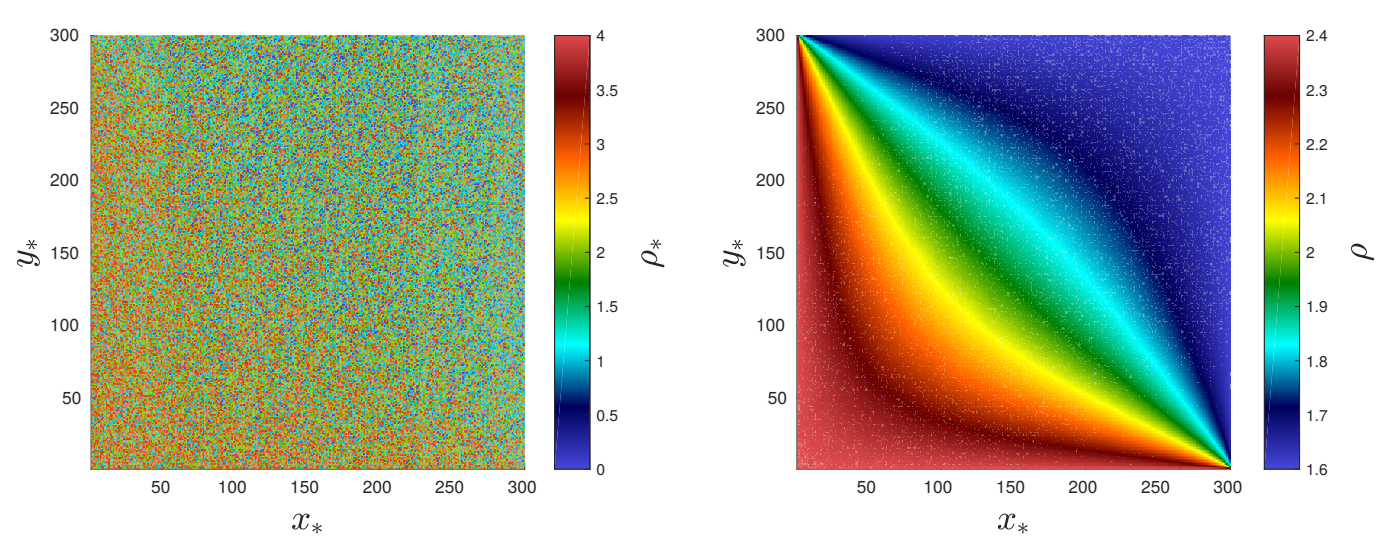

Figure 2. Simulation for $L=300, p=q=1 \rho_{L}=\rho_{B}=2.4$ and $\rho_{R}=\rho_{T}=1$.6. Left: instantaneous microscopic density field in stationary state. Right: average density field measured in stationary state over $10^{6}$ time steps.

We introduce the following macroscopic space and time variables

$$
x=\epsilon x_{*}, \quad y=\epsilon y_{*}, \quad t=\epsilon t_{*}
$$

where $\epsilon=1 / L$. This convective scaling is more relevant to study the coarse-grained dynamics than the diffusive scaling used in the appendix. It can be shown (see Appendix A), that we have the following evolution equation for the average density:

$$
\partial_{t} \rho+\partial_{\alpha} j_{\alpha}=0
$$

with average current (changed by a factor $\epsilon$ compared to the appendix due to the convective scaling).

$$
j_{\alpha}\left(\rho, \partial_{\alpha} \rho\right)=\left(\frac{1}{4}-\frac{4}{q \rho^{2}}\right) \epsilon \partial_{\alpha} \rho .
$$

We note the presence of an anti-diffusive term $\frac{\epsilon}{4} \partial_{\alpha} \rho$ in the expression of the current. This is a spurious term that always appears in the transports coefficients of lattice gas models due to the discrete nature of the dynamics (see, for example, equation 7.4 of [5].). This term is not problematic here since the lattice gas is viewed as a dynamical system that allows illustration of the sub-grid modeling problem while keeping numerical and theoretical analysis simple. The important relevant characteristic of our system is its non-equilibrium nature. We show in figure 2 the microscopic and average density field in stationary state for our simulation: $L=300, p=q=1, \rho_{L}=\rho_{B}=2.4$ and 
$\rho_{R}=\rho_{T}=1.6$ and $10^{6}$ time steps in the statistically stationary state. It is also possible to show that the variance of the microscopic current depends on the average density:

$$
\left\langle\left(j_{* \alpha}-\left\langle j_{* \alpha}\right\rangle\right)^{2}\right\rangle=\frac{\rho}{2}\left(1-\frac{\rho}{4}\right) \equiv \sigma_{*}^{2}(\rho) .
$$

\subsection{Coarse-grained dynamics}

We coarse grain space and time in cubic mesocells of $\tau$ time steps and $\tau \times \tau$ nodes where $\tau$ is called the coarse graining factor. It is therefore possible to define the coarse-grained density $\rho_{\tau}$ and the coarse-grained current $j_{\tau}$ as the average values of the microscopic density and the microscopic current over the mesocell $\mathcal{M}_{\tau}(r, t)=$ $\left\{\left(x_{*}, y_{*}, t_{*}\right) \mid 0 \leq x_{*}-L x<\tau, 0 \leq y_{*}-L y<\tau, 0 \leq t_{*}-L t<\tau\right\}$ at position $r=(x, y)$ and time $t$ :

$$
\begin{aligned}
\tau^{3} \rho_{\tau}(r, t) & =\sum_{\left(r_{*}, t_{*}\right) \in \mathcal{M}_{\tau}(r, t)} \sum_{i=1}^{4} n_{i}\left(r_{*}, t_{*}\right) \\
\tau^{3} j_{\tau \alpha}(r, t) & =\sum_{\left(r_{*}, t_{*}\right) \in \mathcal{M}_{\tau}(r, t)} \sum_{i=1}^{4} n_{i}\left(r_{*}, t_{*}\right) c_{i \alpha} .
\end{aligned}
$$

An instantaneous coarse-grained density field for our simulation with $\tau=5$ is shown in figure 3. Since there is no net separation of scales (i.e. $\tau$ cannot be considered infinite), the fluctuations of the coarse-grained density field are still important.

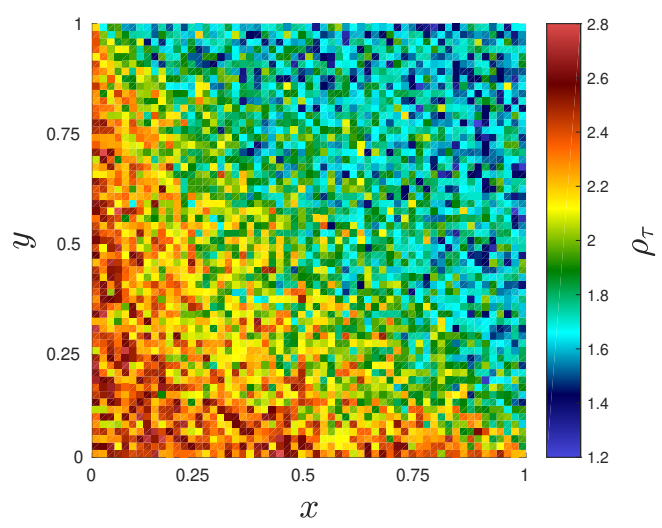

Figure 3. Instantaneous coarse-grained density field for our simulation with a coarse graining factor $\tau=5$.

To simulate the evolution of the coarse-grained field directly, we need a relation that gives the coarse-grained current as a function of the coarse-grained density, its gradient and the coarse graining factor to close the continuity equation

$$
\partial_{t} \rho_{\tau}+\partial_{\alpha} j_{\tau \alpha}=0 .
$$

Since the collisions are local (i.e. involve only particles at one node at each time step), the information does not travel faster than the particles which propagate at a fixed speed 1. It follows that what happen in a mesocell at position $(x, y)$ and a given time $t$ 
only depends on the configurations of the neighbouring mesocells $(x \pm 0, \epsilon \tau, y \pm 0, \epsilon \tau)$ at times $t-\epsilon \tau$. It means that the law governing the evolution of the coarse-grained density is local. Because there is no scale separation, we expect to observe a lag between the coarse-grained current response and the applied coarse-grained density (this rheological property is shared by turbulence and viscoelastic fluids). We therefore have to model the temporal variations of the coarse-grained current. In general, these variations are expected to vary with the coarse-grained density, its gradient, the coarse graining factor, but also the dynamics (i.e. $p$ and $q$ ). Assuming that the temporal variation of one component of the current does not depend on the coarse-grained gradient in the transverse direction, we search a constitutive relation for the current as:

$$
\partial_{t} j_{\tau \alpha}=\mathcal{F}_{\tau \alpha}\left(\rho_{\tau}, \partial_{\alpha} \rho_{\tau}, p, q, \eta\right)
$$

where $\eta$ is the noise representing the sub-mesocell variability.

To this end, we save for each mesocell of the simulation: the coarse-grained current at two successive times and the coarse-grained density measured in the mesocell, and the coarse-grained density of the eight neighbouring mesocells. It allows to compute the quantities

$$
\begin{aligned}
& v_{\tau \alpha}(r, t) \equiv \frac{j_{\tau \alpha}(r, t+\epsilon \tau)-j_{\tau \alpha}(r, t)}{\tau} \quad \alpha=x, y, \\
& g_{\tau x}(r, t) \equiv \frac{\rho_{\tau}(x+\epsilon \tau, y, t)-\rho_{\tau}(x-\epsilon \tau, y, t)}{2 \tau}, \\
& g_{\tau y}(r, t) \equiv \frac{\rho_{\tau}(x, y+\epsilon \tau, t)-\rho_{\tau}(x, y-\epsilon \tau, t)}{2 \tau},
\end{aligned}
$$

which are respectively the first-order approximation of the temporal derivative of the coarse-grained current and the second-order approximation of the spatial derivatives of the coarse-grained density in the microscopic unit. It follows that $v_{\tau \alpha} / \epsilon$ and $g_{\tau \alpha} / \epsilon$ are the finite differences approximations of the spatial derivative in the macroscopic scale estimated with the coarse-grained fields. These are the quantities that need to be modeled in order to perform simulation of the coarse-grained field directly.

\section{Results}

\subsection{Conditional probability distribution of the current}

We define $p_{\tau \alpha}(\hat{v} \mid \hat{j}, \hat{\rho}, \hat{g})$ the conditional PDFs of observing $v_{\tau \alpha} \in\left[\hat{v}-\frac{\mathrm{d} \hat{v}}{2}, \hat{v}+\frac{\mathrm{d} \hat{v}}{2}\right]$ knowing that $j_{\tau \alpha} \in\left[\hat{j}-\frac{\mathrm{d} \hat{j}}{2}, \hat{j}+\frac{\mathrm{d} \hat{j}}{2}\right], \rho_{\tau} \in\left[\hat{\rho}-\frac{\mathrm{d} \hat{\rho}}{2}, \hat{\rho}+\frac{\mathrm{d} \hat{\rho}}{2}\right]$, and $g_{\tau \alpha} \in\left[\hat{g}-\frac{\mathrm{d} \hat{g}}{2}, \hat{g}+\frac{\mathrm{d} \hat{g}}{2}\right]$. In order to identify the structure of these PDFs, it is quite useful to work with normalized random variables. Therefore, we introduce the following random variables:

$$
\delta_{\tau \alpha}(\hat{j}, \hat{\rho}, \hat{g})=\frac{v_{\tau \alpha}-\left\langle v_{\tau \alpha}\right\rangle_{\hat{j}, \hat{\rho}, \hat{g}}}{\sqrt{\left\langle\left(v_{\tau \alpha}-\left\langle v_{\tau \alpha}\right\rangle_{\hat{j}, \hat{\rho}, \hat{g}}\right)^{2}\right\rangle_{\hat{j}, \hat{\rho}, \hat{g}}}} \alpha=x, y, \quad \tau>1
$$


where

$$
\left\langle v_{\tau \alpha}^{n}\right\rangle_{\hat{j}, \hat{\rho}, \hat{g}}=\int \hat{v}^{n} p_{\tau \alpha}(\hat{v} \mid \hat{j}, \hat{\rho}, \hat{g}) \mathrm{d} \hat{v}
$$

is the conditional $n^{t h}$ moment of the variation of the coarse-grained current knowing the coarse-grained density and its gradient.

In figure 4 , we have represented the PDFs of $\delta_{\tau \alpha}(\hat{j}, \hat{\rho}, \hat{g})$ for various values of $\hat{\rho}$, $\hat{g}, \tau=10,15,20,20,30$ and $\alpha=x, y$. We emphasize that the analysis relies on the numerical simulation in the statistically stationary steady-state for $\rho_{L}=\rho_{B}=2.4$, $\rho_{R}=\rho_{T}=1.6$, and $p=q=1$. The histograms are computed dividing $\hat{j}$ into 19 windows between -0.04 and 0.04 , $\hat{\rho}$ divided into 16 windows between 1.2 and 2.8, and $\hat{g}$ divided into 19 windows between -0.03 and 0.03 . The distributions are colored differently depending on $\hat{j}$. We have only retained the samples of size $>2000$ to compute the empirical PDFs in order to limit noise.

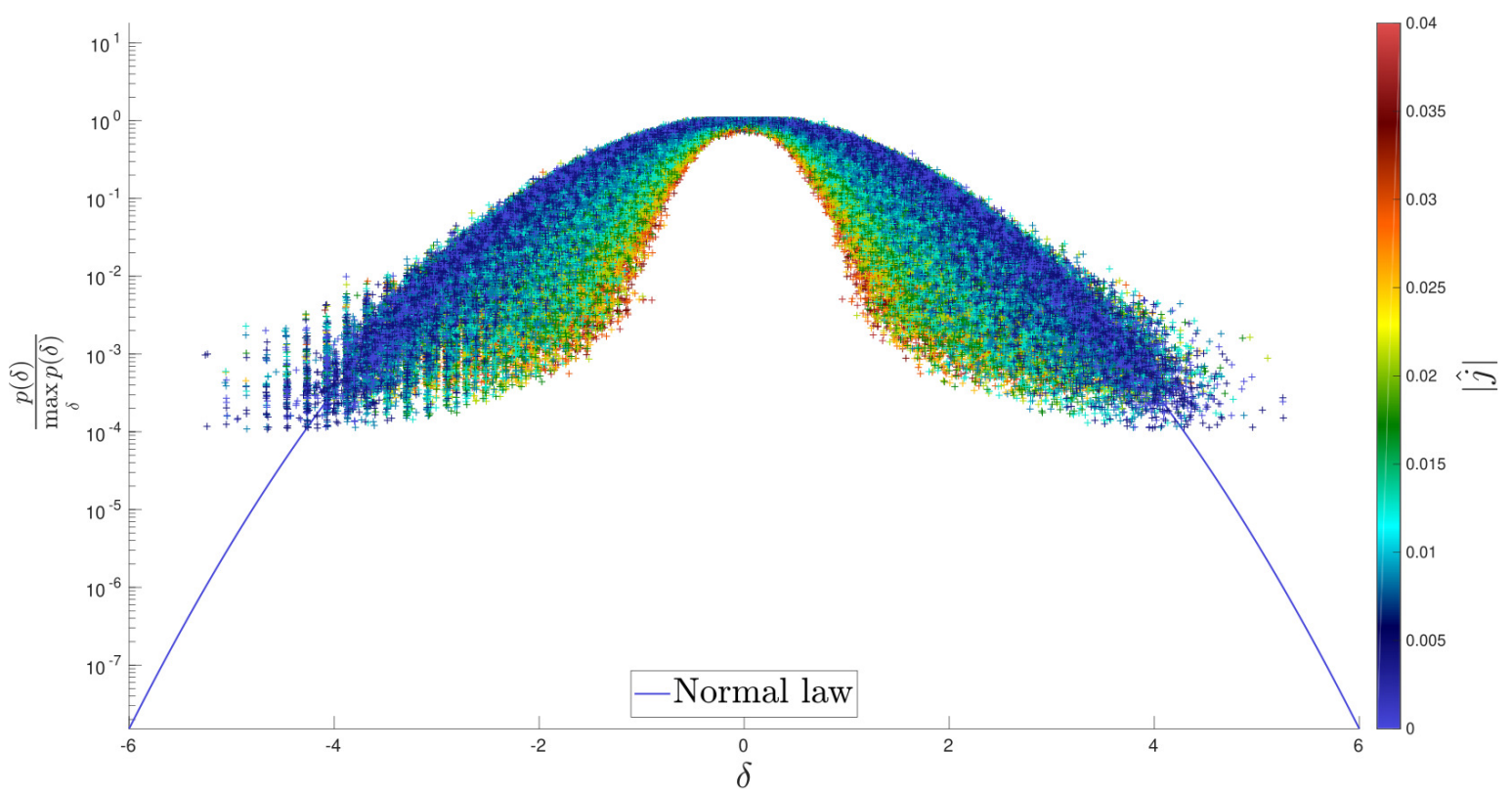

Figure 4. Empirical PDFs of the variables $\delta_{\tau \alpha}$ computed in our simulation for various $\hat{j}, \hat{\rho}, \hat{g}, \tau=10,15,20,25,30$ and $\alpha=x, y$. The Normal law behaviour is indicated by a blue thick line.

For small values of the current $|\hat{j}| \ll 1$ (in blue in figure 4), one observes a universal Normal behavior meaning that $v_{\tau \alpha}$ is a Gaussian random variable fully determined by its mean value and its root mean square (that a priori both depend on the coarse-grained density, its gradient, and the coarse-graining factor) in the limit of low current (or equivalently the low mean speed of particles). We also observe that higher values of the coarse-grained current (in red in figure 4) are associated with a non-Gaussian behavior. This non-Gaussian behavior for high-values of the current is of secondary importance for two reasons. The first is that lattice gas is usually studied in the low-speed limit, 
in particular, to ensure the isotropy of the macroscopic dynamics. The second reason is that the high values of the current are atypical. When we compute the empirical conditional probability distributions of observing $v_{\tau \alpha}$ only knowing $\rho_{\tau}$ and $g_{\tau \alpha}$ (but not $j_{\tau \alpha}$ ), we recover a Gaussian behavior. Then, it is of prime importance to model the coarse-grained dynamics in the low current limit.

The Gaussian behavior leads us to assume that $v_{\tau \alpha}$ can be modelled by the following equation

$$
v_{\tau \alpha}=-\frac{j_{\tau \alpha}-\mu_{\tau \alpha}}{r_{\tau}}+\sigma_{\tau} \eta_{\alpha},
$$

where $\mu_{\tau \alpha}$ is the relaxed current (i.e. in stationary state in the absence of fluctuations), $r_{\tau}$ is the relaxation time, $\sigma_{\tau}$ is the root mean square of $v_{\tau \alpha}$, and $\eta_{\alpha} \alpha=x, y$ are decorrelated normal random variables. Both $\mu_{\tau \alpha}, r_{\tau}$ and $\sigma_{\tau}$ have to be modelled to construct the sub-grid model. If they are considered as constant, the Gaussian behavior will follow. Note however that if we allow $\sigma_{\tau}$ and $r_{\tau}$ to be statistical variables, we could get a non-Gaussian solution of equation (18) as a superstatistics [19]. In the sequel, however, we concentrate on low-current modeling and assume that $\sigma_{\tau}$ and $r_{\tau}$ are deterministic functions that need to be modeled. For a given value of $\rho_{\tau}$ and $g_{\tau \alpha}$, the linear regression for the points $v_{\tau \alpha}$ vs $j_{\tau \alpha}$ has a slope $-1 / r_{\tau}$, an intercept $\mu_{\tau \alpha} / r_{\tau}$ and an error term $\sigma_{\tau}$. This is how we estimate $\mu_{\tau \alpha}, r_{\tau}$ and $\sigma_{\tau}$ numerically. In the following, we discuss the behaviour of these functions with $\rho_{\tau}, g_{\tau \alpha}, q$ and $\tau$.

\subsection{Relaxed current $\mu_{\tau \alpha}$}

The figure 5 shows $\mu_{\tau \alpha}$ for $\tau=10$ in our simulation and the average current predicted in the hydrodynamic limit with a convective scaling (given by equation (7)). We do not show the results for other values of $\tau$ because our statistics are not sufficient to provide a converged estimate of $\mu_{\tau \alpha}$ for large values of $g_{\tau \alpha}$. Quite remarkably, we observe that the coarse-grained current tends to relax to the average current (obtained in the hydrodynamic limit) estimated by the local coarse-grained density and its gradient. It means that the relaxed current is invariant under the effect of coarse graining. This suggests that the relaxed coarse-grained current obeys the same functional relation as a function of the (coarse-grained) gradient density than the microscopic gradient, a property that can be viewed as a kind of Germano identity [20]. In turbulence, such identity is used via a hypothesis of scale invariance of the turbulence in the inertial range, resulting in a Reynolds stress that keeps the same shape above and below the cut-off. In our case, the Germano identity amounts to take

$$
\mu_{\tau \alpha}\left(\rho_{\tau}, g_{\tau \alpha}, q\right)=\left(\frac{1}{4}-\frac{4}{q \rho_{\tau}^{2}}\right) g_{\tau \alpha}
$$

for our sub-grid model. 

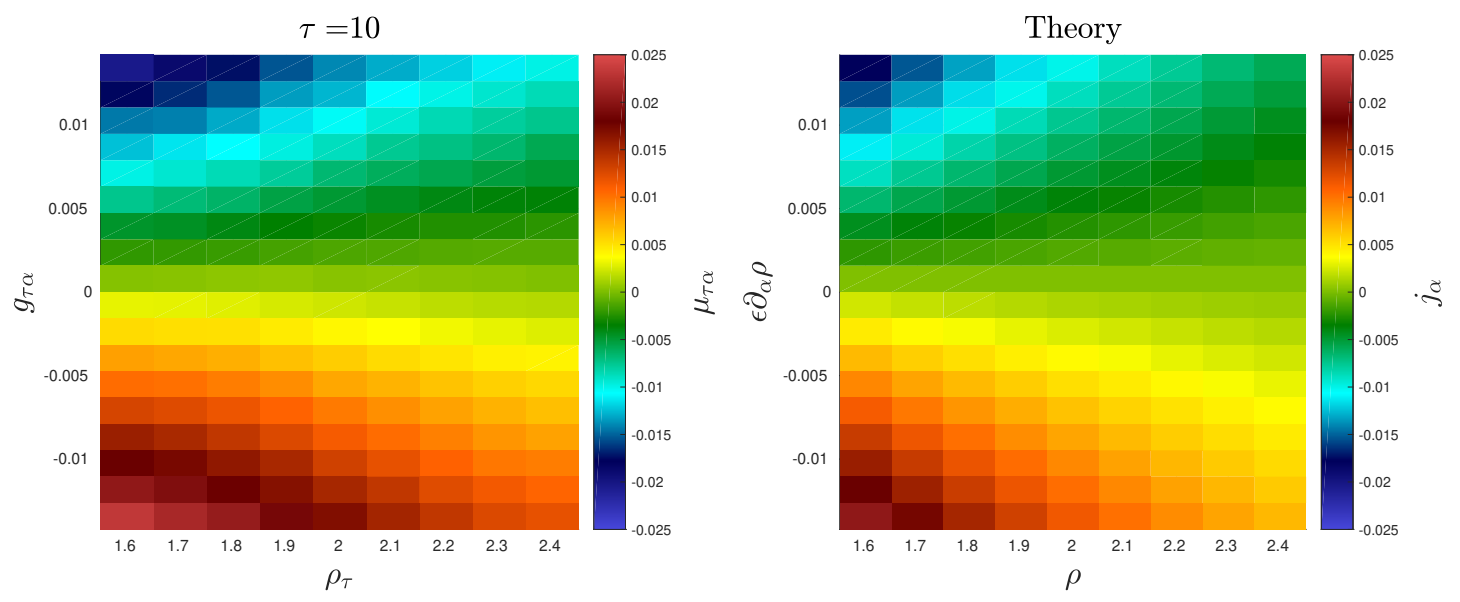

Figure 5. Left: relaxed coarse-grained current $\mu_{\tau \alpha}$ as a function of the coarse-grained density and its gradient for $\tau=10$ in our simulation. Right: theoretical relation obtained by the Chapman-Enskog expansion for the averaged observables.

\subsection{Relaxation time $r_{\tau}$ and typical fluctuations $\sigma_{\tau}$}

We show on figure 6 the relaxation time (divided by the coarse graining factor) and the root mean square of $v_{\tau \alpha}$ as a function of the coarse-grained density and its gradient for $\tau=10$.
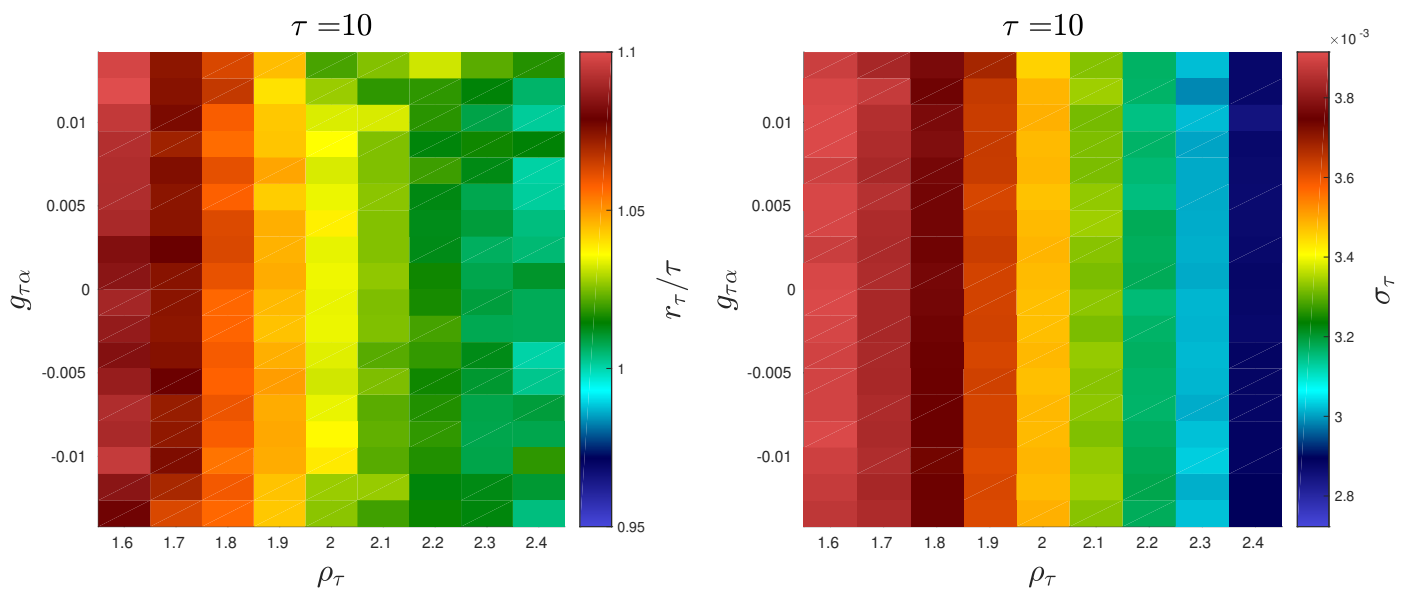

Figure 6. Relaxation time $r_{\tau}$ divided by the coarse graining factor (left) and the root mean square of the variation of the coarse-grained current $\sigma_{\tau}$ (right) as functions of the coarse-grained density and its gradient for $\tau=10$ in our simulation.

We observe that both $r_{\tau}$ and $\sigma_{\tau}$ depend little on the gradient of the coarse-grained density (at least for the range of parameter we have investigated), suggesting that the relaxation and the fluctuations of $v_{\tau \alpha}$ can be modelled by the equilibrium fluctuations at the coarse-grained size at a first approximation. We propose a simple model where we simplify the dynamics by considering that at each discrete time step, the mesocell 
Sub-grid modelling of a diffusive lattice gas

Table 1. Our model for the gain/loss $X=-1,0,1$ of particle with speed $c_{1}$ at a given time at each node in the mesocell for the population $N_{s 1}$.

\begin{tabular}{lll}
\hline Source/Sink & nodes & probability distribution \\
\hline entry (left boundary) & $\tau$ & $\left\{\begin{array}{l}P(X=-1)=0, \\
P(X=0)=1-\rho_{\tau} / 4, \\
P(X=1)=\rho_{\tau} / 4 .\end{array}\right.$ \\
\hline exit (right boundary) & $\tau$ & $\left\{\begin{array}{l}P(X=-1)=N_{s 1}, \\
P(X=0)=1-N_{s 1}, \\
P(X=1)=0 .\end{array}\right.$ \\
\hline 2 particules collision & $\tau^{2} \quad\left\{\begin{array}{l}P(X=-1)=N_{s 1}\left(1-N_{s 2}\right) N_{s 3}\left(1-N_{s 4}\right) p, \\
P(X=0)=1-P(X=-1)-P(X=1), \\
P(X=1)=\left(1-N_{s 1}\right) N_{s 2}\left(1-N_{s 3}\right) N_{s 4} p .\end{array}\right.$ \\
\hline 3 particules collision & $\tau^{2} \quad\left\{\begin{array}{l}P(X=-1)=N_{s 1} N_{s 2}\left(1-N_{s 3}\right) N_{s 4} q, \\
P(X=0)=1-P(X=-1)-P(X=1), \\
P(X=1)=\left(1-N_{s 1}\right) N_{s 2} N_{s 3} N_{s 4} q .\end{array}\right.$ \\
\hline
\end{tabular}

is spatially statistically homogeneous (i.e. we neglect the spatial inhomogeneities at all time step of the mesocell). This crude approximation allows to describe the state of the box at each time step by the mean occupations of channels in the mesocell, noted $N_{s i}\left(t_{*}\right), i=1, \ldots, 4$. It implicitly assumes an instantaneous mixing of particles at each discrete time step inside the bulk of the mesocell. To write the evolution equation for the $N_{s i}$ 's, one must distinguish the surface effects (rate at which particles enter/leave the box), and the volume effect due to collisions. Naturally, the scalings of these processes are different since they do not involve the same number of nodes. We model the variations of the populations at each time step by considering the different sources and sinks as stochastic processes. For a given population $i$, particles enter at a boundary with a rate $\rho_{\tau} / 4$ at each node. They exit the mesocells at the opposite boundary with a rate $N_{s i}$ at each node. By doing so, we implicitly neglect the collision step at boundaries. Particles populations are mixed by collisions that occur in the all bulk. It is taken into account by considering the effect of collisions of the mean population at one node. For example, the sources/sinks for $N_{s 1}$ are given in table 1 .

Considering $\tau$ to be sufficiently large, we can approximate the random change of populations with Gaussian random variables using the central limit theorem. For example, the resulting stochastic equation describing the evolution of $N_{s 1}$ is

$$
\begin{gathered}
\tau^{2}\left(N_{s 1}\left(t_{*}+1\right)-N_{s 1}\left(t_{*}\right)\right)=\tau \frac{\rho_{\tau}}{4}+\sqrt{\tau \frac{\rho_{\tau}}{4}\left(1-\frac{\rho_{\tau}}{4}\right)} \eta_{1}^{\text {in }}-\tau N_{s 1}-\sqrt{\tau N_{s 1}\left(1-N_{s 1}\right)} \eta_{1}^{\text {out }} \\
+\tau^{2} p N_{p}+\sqrt{\tau^{2} \sigma_{p}^{2}} \eta_{p}+\tau^{2} q N_{q_{x}}+\sqrt{\tau^{2} \sigma_{q_{x}}^{2}} \eta_{q_{x}}
\end{gathered}
$$

where $\eta_{1}^{\text {in }}, \eta_{1}^{\text {out }}, \eta_{p}$ and $\eta_{q_{x}}$ are all independents, identically distributed normal random 
variables, and

$$
\begin{aligned}
& N_{p}=\left(1-N_{s 1}\right) N_{s 2}\left(1-N_{s 3}\right) N_{s 4}-N_{s 1}\left(1-N_{s 2}\right) N_{s 3}\left(1-N_{s 4}\right), \\
& \sigma_{p}^{2}=\left(1-N_{s 1}\right) N_{s 2}\left(1-N_{s 3}\right) N_{s 4} p+N_{s 1}\left(1-N_{s 2}\right) N_{s 3}\left(1-N_{s 4}\right) p-\left(p N_{p}\right)^{2}, \\
& N_{q_{x}}=\left(1-N_{s 1}\right) N_{s 2} N_{s 3} N_{s 4}-N_{s 1} N_{s 2}\left(1-N_{s 3}\right) N_{s 4}, \\
& \sigma_{q_{x}}^{2}=\left(1-N_{s 1}\right) N_{s 2} N_{s 3} N_{s 4} q+N_{s 1} N_{s 2}\left(1-N_{s 3}\right) N_{s 4} q-\left(q N_{q_{x}}\right)^{2} .
\end{aligned}
$$

Similarly, one can write down the equation for $N_{s 3}$

$$
\begin{gathered}
\tau^{2}\left(N_{s 3}\left(t_{*}+1\right)-N_{s 3}\left(t_{*}\right)\right)=\tau \frac{\rho_{\tau}}{4}+\sqrt{\tau \frac{\rho_{\tau}}{4}\left(1-\frac{\rho_{\tau}}{4}\right)} \eta_{3}^{\text {in }}-\tau N_{s 3}-\sqrt{\tau N_{s 3}\left(1-N_{s 3}\right)} \eta_{3}^{\text {out }} \\
+\tau^{2} p N_{p}+\sqrt{\tau^{2} \sigma_{p}^{2}} \eta_{p}-\tau^{2} q N_{q_{x}}-\sqrt{\tau^{2} \sigma_{q_{x}}^{2}} \eta_{q_{x}} .
\end{gathered}
$$

We point out that $\eta_{3}^{\text {in }}$ and $\eta_{3}^{\text {out }}$ are also identically distributed normal random variables, and that $\eta_{p}$ and $\eta_{q_{x}}$ are the same random variables involved in (20) due to the conservation of the number of particles in the mesocell during the collision step.

Now, we introduce the spatially coarse-grained current (over a square mesocell) in the $x$ direction $j_{s x} \equiv N_{s 1}-N_{s 3}$. One obtains the evolution equation for $j_{s x}$ by taking the difference of (20) and (25), divided by $\tau^{2}$ :

$$
\begin{aligned}
& j_{s x}\left(t_{*}+1\right)-j_{s x}\left(t_{*}\right)=-\frac{1}{\tau} j_{s x}+2 q N_{q_{x}}+\sqrt{\frac{1}{\tau^{3}} \frac{\rho_{\tau}}{4}\left(1-\frac{\rho_{\tau}}{4}\right)}\left(\eta_{1}^{\text {in }}-\eta_{3}^{\text {in }}\right) \\
& -\sqrt{\frac{1}{\tau^{3}} N_{s 1}\left(1-N_{s 1}\right)} \eta_{1}^{\text {out }}+\sqrt{\frac{1}{\tau^{3}} N_{s 3}\left(1-N_{s 3}\right)} \eta_{3}^{\text {out }}+2 \sqrt{\frac{1}{\tau^{2}} \sigma_{q_{x}}^{2}} \eta_{q_{x}} .
\end{aligned}
$$

To simplify the last equation, we linearise $N_{q_{x}}$ around the mean occupation of channels $\rho_{\tau} / 4: N_{s i}\left(t_{*}\right) \simeq \rho_{\tau} / 4+\delta N_{s i}\left(t_{*}\right), i=1, \ldots, 4$ with $\delta N_{s i}\left(t_{*}\right)<<1$. Moreover, we consider only the additive part of the noises. We therefore evaluate

$$
\begin{aligned}
& N_{q_{x}} \simeq \frac{\rho_{\tau}^{2}}{16}\left(\delta N_{s 3}-\delta N_{s 1}\right)=-\frac{\rho_{\tau}^{2}}{16} j_{s x}, \\
& \sqrt{N_{s i}\left(1-N_{s i}\right)} \simeq \sqrt{\frac{\rho_{\tau}}{4}\left(1-\frac{\rho_{\tau}}{4}\right)}, \quad i=1, \ldots, 4 \\
& \sigma_{q_{x}}^{2} \simeq 2 q\left(\frac{\rho_{\tau}}{4}\right)^{3}\left(1-\frac{\rho_{\tau}}{4}\right) .
\end{aligned}
$$

It follows that

$$
\begin{gathered}
j_{s x}\left(t_{*}+1\right)-j_{s x}\left(t_{*}\right)=-\left(\frac{1}{\tau}+\frac{q}{8} \rho_{\tau}^{2}\right) j_{s x}+\sqrt{\frac{1}{\tau^{3}} \frac{\rho_{\tau}}{4}\left(1-\frac{\rho_{\tau}}{4}\right)}\left(\eta_{1}^{\text {in }}-\eta_{1}^{\text {out }}-\eta_{3}^{\text {in }}+\eta_{3}^{\text {out }}\right) \\
+\sqrt{\frac{1}{\tau^{2}} \frac{q}{8} \rho_{\tau}^{3}\left(1-\frac{\rho_{\tau}}{4}\right)} \eta_{q_{x} .}
\end{gathered}
$$

Now, we use basic properties of independent Gaussian random variables to write this equation in a more compact form:

$j_{s x}\left(t_{*}+1\right)-j_{s x}\left(t_{*}\right)=-\left(\frac{1}{\tau}+\frac{q}{8} \rho_{\tau}^{2}\right) j_{s x}+\sqrt{\frac{1}{\tau^{2}} \rho_{\tau}\left(1-\frac{\rho_{\tau}}{4}\right)\left(\frac{1}{\tau}+\frac{q}{8} \rho_{\tau}^{2}\right)} \eta_{x}$

where $\eta_{x}$ is a normal random variable. The equation (31) is a Langevin equation that arise in many contexts in Physics (see for example [21] for an introduction to stochastic 
methods). The large deviation function of the equivalent of the time integrated current in our model, given by the limit for $\tau \rightarrow \infty$ of

$$
j_{\tau x}(t)=\frac{1}{\tau} \sum_{t \leq \epsilon t_{*}<t+\epsilon \tau} j_{s x}\left(t_{*}\right)
$$

has already been computed for this process (see, for example, the last section of [22]). One can deduce from these results that the distribution of $j_{\tau x}$ satisfies a large deviation principle

$$
\lim _{\tau \rightarrow \infty}-\frac{1}{\tau} \ln P\left(j_{\tau x}=j\right)=I(j)
$$

where the rate function is given by

$$
I(j)=\frac{1}{2} \frac{\left(\frac{1}{\tau}+\frac{q}{8} \rho_{\tau}^{2}\right)^{2}}{\frac{1}{\tau^{2}} \rho_{\tau}\left(1-\frac{\rho_{\tau}}{4}\right)\left(\frac{1}{\tau}+\frac{q}{8} \rho_{\tau}^{2}\right)} j^{2}=\frac{\tau^{2}}{2} \frac{\left(\frac{1}{\tau}+\frac{q}{8} \rho_{\tau}^{2}\right)}{\rho_{\tau}\left(1-\frac{\rho_{\tau}}{4}\right)} j^{2} .
$$

Therefore, our model states that $j_{\tau \alpha}$ is a Gaussian random variable with mean zero and root mean square $\sqrt{\frac{\rho_{\tau}\left(1-\frac{\rho_{\tau}}{4}\right)}{\tau^{3}\left(\frac{1}{\tau}+\frac{q}{8} \rho_{\tau}^{2}\right)}}$. We suggest that $v_{\tau \alpha}$ fluctuates like $j_{\tau \alpha} / \tau$. It is equivalent to choose

$$
\sigma_{\tau}\left(\rho_{\tau}, q\right)=\sqrt{\frac{\rho_{\tau}\left(1-\frac{\rho_{\tau}}{4}\right)}{\tau^{5}\left(\frac{1}{\tau}+\frac{q}{8} \rho_{\tau}^{2}\right)}}
$$

for our sub-grid model. In figure 7 , we compare this model to the numerical results. The
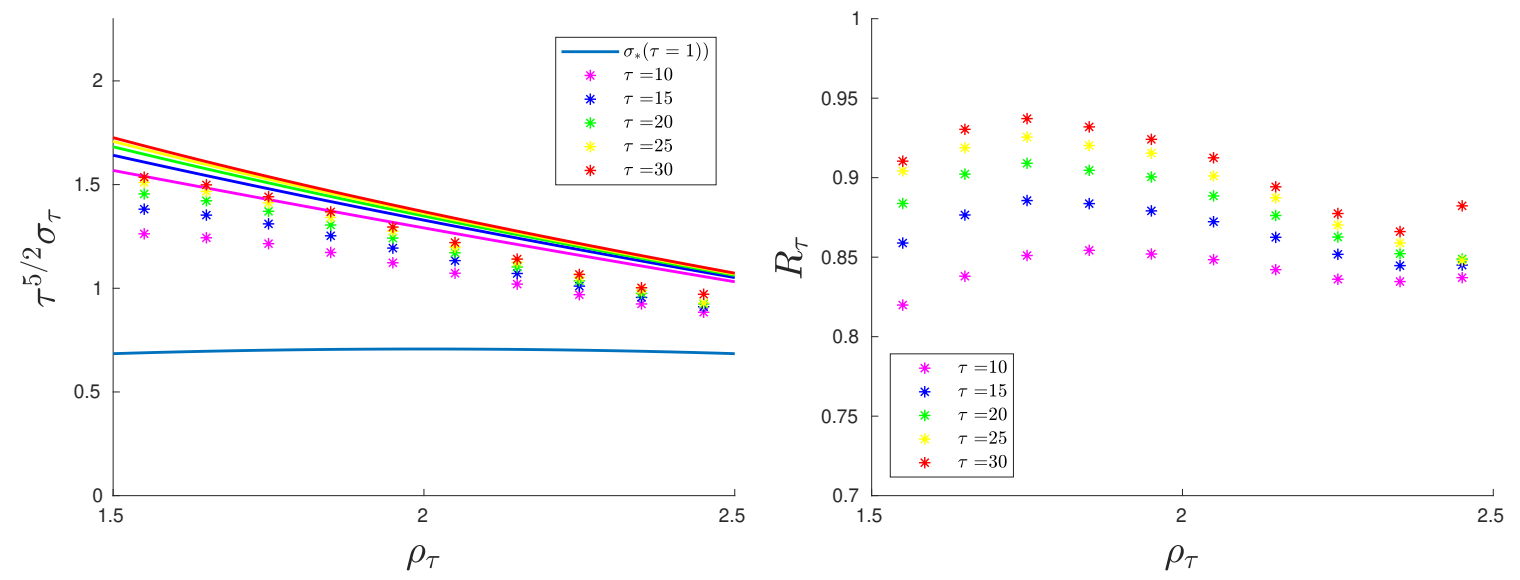

Figure 7. Left: $\sigma_{\tau}$ multiplied by $\tau^{5 / 2}$ as a function of the coarse-grained density. The model (35) (in full lines) and the root mean square of the microscopic current (in light blue full line) are compared to the numerical results (in stars) for various values of $\tau$. Right: Ratio between the numerical value of $\sigma_{\tau}$ and our model $R_{\tau}$.

numerical results show that as far as we coarse grain, we change the shape and the scaling of the fluctuations of the coarse-grained current as a function of the coarse-grained density. Strikingly, $\sigma_{\tau}$ is no more symmetric around the density $\rho_{\tau}=2$. It goes from $\sqrt{\frac{\rho}{2}\left(1-\frac{\rho}{4}\right)}$ for the microscopic fluctuations (corresponding to $\tau=1$ ) to a limit function that we have not attained with our limited simulation. Our model provides the correct 
variation of $\sigma_{\tau}(\rho)$ with respect to $(\rho \simeq 2)$. To quantify the relative error, we compute the ratio between the numerical value of $\sigma_{\tau}$ and our theoretical prediction noted $R_{\tau}$. It is provided in figure 7. We observe that the variations of $R_{\tau}$ with $\rho_{\tau}$ are rather mild, and that the deviations between the simulation result and the model remain within about $10 \%$ for sufficiently large $\tau$ (in the regime of $1.5<\rho_{r}<2.0$ ). This suggests that observed deviations from our theoretical modeling are finite-size effects.

Averaging the equation (31), the evolution of $\left\langle j_{s x}\right\rangle$ in the mesocell can be written

$$
\left\langle j_{s x}\right\rangle\left(t_{*}+1\right)=a\left\langle j_{s x}\right\rangle\left(t_{*}\right), \quad a=1-\left(\frac{1}{\tau}+\frac{q}{8} \rho_{\tau}^{2}\right) .
$$

It yields to the relation

$$
\left\langle j_{s x}\right\rangle\left(t_{*}^{\prime}\right)=\left\langle j_{s x}\right\rangle\left(t_{*}\right) a^{t_{*}^{\prime}-t_{*}} \quad \forall t_{*}, t_{*}^{\prime} \in \mathbb{N} .
$$

Using the last relation in the average of equation (32), we show that

$$
\left\langle j_{\tau x}\right\rangle(t+\epsilon \tau)=a^{\tau}\left\langle j_{\tau x}\right\rangle(t) .
$$

It follows that

$$
\frac{\left\langle j_{\tau x}\right\rangle(t+\epsilon \tau)-\left\langle j_{\tau x}\right\rangle(t)}{\tau}=-\frac{1-a^{\tau}}{\tau}\left\langle j_{\tau x}\right\rangle(t) .
$$

This equation suggests that the relaxation time for the coarse-grained current is

$$
r_{\tau}\left(\rho_{\tau}, q\right)=\frac{\tau}{1-a^{\tau}}=\frac{\tau}{1-\left(1-\left(\frac{1}{\tau}+\frac{q}{8} \rho_{\tau}^{2}\right)\right)^{\tau}} .
$$

Since $-1-\frac{1}{\tau}=a\left(\rho_{\tau}=4\right) \leq a \leq\left(\rho_{\tau}=0\right)=1-\frac{1}{\tau}$, we expect that in normal conditions $\left(\rho_{\tau}\right.$ not too close from 4$)|a|<1$ so $a^{\tau} \rightarrow 0$ when $\tau \rightarrow \infty$. We observe on figure 8 that this model correctly predicts that the relaxation time converges to $\tau$ as $\tau$ increases. The agreement between the prediction and the model is better than 10 percent.

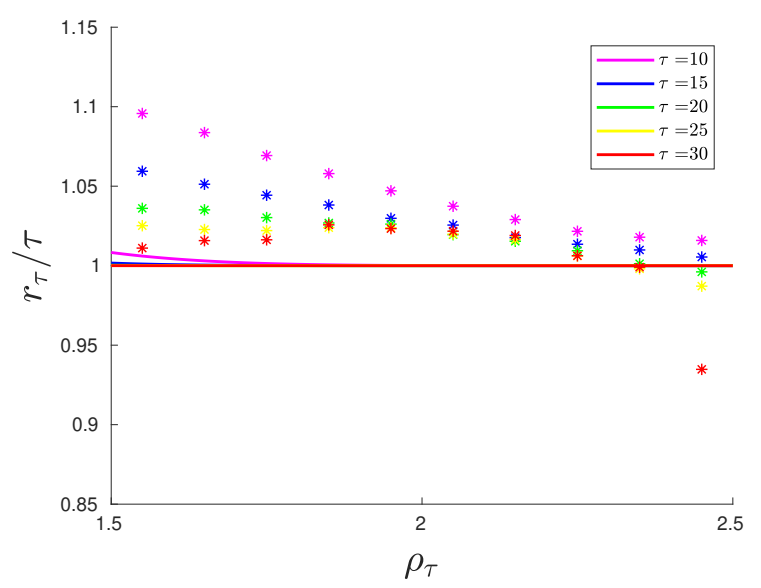

Figure 8. Relaxation time $r_{\tau}$ divided by $\tau$ for our simulation with various values of $\tau$ (represented with stars) compared to our model (40) (represented with full lines). 


\section{Discussion and conclusion}

In this study, we have addressed the question of sub-grid modelling in a simple diffusive Lattice Gas. In the low current limit, we observed that the first order approximation of the temporal derivative of the coarse-grained current, knowing the coarse-grained density and its gradient, is a Gaussian random variable whose mean and root mean square depend only on the size of the mesocell, the coarse-grained density, its gradient, and eventually on the dynamical parameters of the model. We have proposed a simple model where the temporal variation of the coarse-grained current is modelled as the sum of an average relaxation to a relaxed current at a given relaxation time, and a Gaussian noise. Observing the fact that the root mean square and the relaxation time depend little on the gradient of density, we have constructed a simple equilibrium model where the spatial inhomogeneities inside a mesocell are neglected to determine the variations of the root mean square of the grained current and the relaxation time. Our model agrees quantitatively with the numerical results, providing a agreement better that 20 percent for the fluctuations, and better than 10 percent for the relaxation time. In particular, it explains the variations of the relaxation time and the root mean square as a function of the coarse-grained density. The relaxed current is simply given by taking the hydrodynamic limit.

LGCA and SLG have been studied extensively as simplified model of kinetic theory [8] or paradigmatic models of non-equilibrium physics [9]. However, up to our knowledge, these models are not used as conceptual object to learn about sub-grid modelling (we can note one attempt to compute a turbulent viscosity with such model [23]). Yet, deriving sub-grid models for Lattice Gases seems to be an interesting problem to deal with before addressing more difficult problems like the turbulence modelling. In particular to understand the non-trivial variations of the coefficients that are introduced empirically in turbulence models. As an example, our simple model for the fluctuations of the current shows that the shape of the root mean square of the coarse-grained current (as a function of the density) is changed when we coarse grain.

Our model for the evolution of the coarse-grained density is finally

$$
\begin{aligned}
& \partial_{t} \rho_{\tau}+\partial_{\alpha} j_{\tau \alpha}=0, \\
& \epsilon \partial_{t} j_{\tau \alpha}=-\frac{j_{\tau \alpha}-\mu_{\tau \alpha}}{r_{\tau}}+\sigma_{\tau} \eta_{\alpha},
\end{aligned}
$$

where $\epsilon=1 / L$ is the ratio between the microscopic scale and the size of the system, $\eta_{\alpha}$ is a Gaussian white noise and $\mu_{\tau \alpha}, r_{\tau}$ and $\sigma_{\tau}$ are specified in equations (19), (40) and (35).

For this diffusive lattice gas, there remains the question of the non-Gaussian fluctuations observed for the atypical large values of the coarse-grained current. We expect that a refined sub-grid model (that takes into account the spatial inhomogeneities in-

side the mesocell) can be constructed by extending recent works on SLG (like for the 
Zero Range Process [24]) to compute the statistics of coarse-grained observables, taking into account fluctuations at boundaries of the mesocell. Alternatively, one could use the superstatistics formalism to derive more elaborate sub-grid models $[19,25,26]$. It would also be interesting to perform simulations with different boundary conditions, for different values of the dynamical parameters $p$ and $q$, and for transient simulation to test the robustness of the sub-grid model to various conditions.

An important question is whether one can employ such a method to other systems of practical interest, like turbulent flows. For example, does a relaxation equation similar to the one used here could be used to model the Reynolds stress tensor using the analysis of the coarse-grained velocity field? To our knowledge, such Eulerian model has not been tested for other systems. 
Sub-grid modelling of a diffusive lattice gas

\section{Acknowledgments}

This work has been supported by the ANR EXPLOIT, grant agreement no. ANR-16CE06-0006-01. We thank the anonymous referees for their useful comments that have helped to improve significantly the article.

\section{Appendix A. Hydrodynamic limit and microscopic fluctuations of the current}

Appendix A.1. Average current

For a LGCA (i.e. for a given lattice and collision rules), one can derive the macroscopic behaviour of observables at large scales via the equation verified by the average quantities (here $\rho$ and $j$ ) in the limit of infinite lattice size. The most popular way to it is to apply the multi-scale analysis used in the continuous kinetic theory (the so-called Chapman-

Enskog expansion) to the discrete lattice gas. Here, a simple diffusive scaling is sufficient to derive the macroscopic equation for the evolution of the average density. To do this, we start with the local micro-dynamic equation for the Boolean field which is given by the Chapman-Kolmogorov equation [5, 8]. For our simple model with only four velocities, we can explicitly write it:

$n_{1}\left(x_{*}+1, y_{*}, t_{*}+1\right)=n_{1}\left(x_{*}, y_{*}, t_{*}\right)+\zeta_{p} n_{p}\left(x_{*}, y_{*}, t_{*}\right)+\zeta_{q_{x}} n_{q_{x}}\left(x_{*}, y_{*}, t_{*}\right)$,
$n_{2}\left(x_{*}, y_{*}+1, t_{*}+1\right)=n_{2}\left(x_{*}, y_{*}, t_{*}\right)-\zeta_{p} n_{p}\left(x_{*}, y_{*}, t_{*}\right)+\zeta_{q_{y}} n_{q_{y}}\left(x_{*}, y_{*}, t_{*}\right)$,
$n_{3}\left(x_{*}-1, y_{*}, t_{*}+1\right)=n_{3}\left(x_{*}, y_{*}, t_{*}\right)+\zeta_{p} n_{p}\left(x_{*}, y_{*}, t_{*}\right)-\zeta_{q_{x}} n_{q_{x}}\left(x_{*}, y_{*}, t_{*}\right)$,
$n_{4}\left(x_{*}, y_{*}-1, t_{*}+1\right)=n_{4}\left(x_{*}, y_{*}, t_{*}\right)-\zeta_{p} n_{p}\left(x_{*}, y_{*}, t_{*}\right)-\zeta_{q_{y}} n_{q_{y}}\left(x_{*}, y_{*}, t_{*}\right)$,

with

$$
\begin{aligned}
& n_{p}=\left(1-n_{1}\right) n_{2}\left(1-n_{3}\right) n_{4}-n_{1}\left(1-n_{2}\right) n_{3}\left(1-n_{4}\right), \\
& n_{q_{x}}=\left(1-n_{1}\right) n_{2} n_{3} n_{4}-n_{1} n_{2}\left(1-n_{3}\right) n_{4}, \\
& n_{q_{y}}=n_{1}\left(1-n_{2}\right) n_{3} n_{4}-n_{1} n_{2} n_{3}\left(1-n_{4}\right),
\end{aligned}
$$

where $\zeta_{p}\left(\zeta_{q_{x}}\right.$ or $\left.\zeta_{q_{y}}\right)$ represents a Bernoulli random variables with mean $p(q)$, that are decorrelated in space and time, and with the $n_{i}$ 's. We have omitted the spatio-temporal dependence $\left(\zeta\left(x_{*}, y_{*}, t_{*}\right) \rightarrow \zeta\right.$ and $\left.n_{i}\left(x_{*}, y_{*}, t_{*}\right) \rightarrow n_{i}\right)$ in the three last equations for brevity.

We now use three assumptions:

(i) The lattice Boltzmann (Mean Field) approximation: the correlations between particles before collisions are neglected. This allows to write

$$
\left\langle\prod_{i \in I} n_{i}\left(r_{*}, t_{*}\right)\right\rangle=\prod_{i \in I}\left\langle n_{i}\left(r_{*}, t_{*}\right)\right\rangle \quad \forall r_{*} \in \mathcal{L}, t_{*} \in \mathbb{N},
$$

where $I$ is any set of non repeated indices of the channels. 
(ii) The Hydrodynamic limit consists to introduce the (dimensionless) variables

$$
t=t_{*} / T, \quad x=x_{*} / L, \quad y=y_{*} / L,
$$

where $T \rightarrow \infty$ and $L \rightarrow \infty$ are the macroscopic time and space (typically the time of the simulation and the size of the Lattice), such that $t, r=(x, y)$ are considered as continuous variables. We then introduce a macroscopic population that varies continuously in space and time

$$
f_{i}(r, t)=\left\langle n_{i}\left(r_{*}, t_{*}\right)\right\rangle=N_{i}\left(r_{*}, t_{*}\right) .
$$

We keep the same notation for the continuous density and current. In the following, we will consider the diffusive scaling $T=L^{2}$ and introduce the Knudsen number $\epsilon=1 / L \rightarrow 0$.

(iii) We can show that the equilibrium solution (i.e. the solution in stationary state without perturbation) of the system is $f_{i}^{(0)}=\rho / 4$ (we can invoke the Universality Theorem for lattice gases, valid in case of semi-detailed balance and no spurious invariant, in the simple case where only mass is conserved by collisions $[5,8])$. We assume small perturbations around this equilibrium such that

$$
f_{i}=\frac{\rho}{4}+\epsilon f_{i}^{\prime}, \quad \sum_{i=1}^{4} f_{i}^{\prime}=0 .
$$

Averaging (A.1-A.4), using (A.8), substituting (A.11) in (A.5-A.7) and replacing the finite difference by derivatives up to second order in $\epsilon$ with the diffusive scaling, we obtain

$$
\begin{aligned}
\left(\epsilon \partial_{x}+\epsilon^{2} \partial_{t}+\epsilon^{2} \frac{1}{2} \partial_{x}^{2}\right)\left(\frac{\rho}{4}+\epsilon f_{1}^{\prime}\right) & =\epsilon p \frac{\rho}{4}\left(1-\frac{\rho}{4}\right)\left(f_{2}^{\prime}+f_{4}^{\prime}-f_{1}^{\prime}-f_{3}^{\prime}\right) \\
& +\epsilon^{2} p\left(1-\frac{\rho}{2}\right)\left(f_{2}^{\prime} f_{4}^{\prime}-f_{1}^{\prime} f_{3}^{\prime}\right) \\
+ & \epsilon q\left(f_{3}^{\prime}-f_{1}^{\prime}\right) \frac{\rho^{2}}{16} \\
+ & \epsilon q \frac{\rho}{4}\left(f_{3}^{\prime}-f_{1}^{\prime}\right)\left(f_{2}^{\prime}+f_{4}^{\prime}\right)+O\left(\epsilon^{3}\right), \\
\left(-\epsilon \partial_{x}+\epsilon^{2} \partial_{t}+\epsilon^{2} \frac{1}{2} \partial_{x}^{2}\right)\left(\frac{\rho}{4}+\epsilon f_{3}^{\prime}\right) & =\epsilon p \frac{\rho}{4}\left(1-\frac{\rho}{4}\right)\left(f_{2}^{\prime}+f_{4}^{\prime}-f_{1}^{\prime}-f_{3}^{\prime}\right) \\
& +\epsilon^{2} p\left(1-\frac{\rho}{2}\right)\left(f_{2}^{\prime} f_{4}^{\prime}-f_{1}^{\prime} f_{3}^{\prime}\right) \\
& -\epsilon q\left(f_{3}^{\prime}-f_{1}^{\prime}\right) \frac{\rho^{2}}{16} \\
& -\epsilon^{2} q \frac{\rho}{4}\left(f_{3}^{\prime}-f_{1}^{\prime}\right)\left(f_{2}^{\prime}+f_{4}^{\prime}\right)+O\left(\epsilon^{3}\right) .
\end{aligned}
$$

Here and in the following, we only treat $f_{1}^{\prime}$ and $f_{3}^{\prime}$, similar equations hold $f_{2}^{\prime}$ and $f_{4}^{\prime}$. Recasting the terms of the same order in $\epsilon$, one has

$$
\begin{aligned}
& \epsilon^{1}: \\
& \partial_{x} \frac{\rho}{4}=p \frac{\rho}{4}\left(1-\frac{\rho}{4}\right)\left(f_{2}^{\prime}+f_{4}^{\prime}-f_{1}^{\prime}-f_{3}^{\prime}\right)+q\left(f_{3}^{\prime}-f_{1}^{\prime}\right) \frac{\rho^{2}}{16},
\end{aligned}
$$


Sub-grid modelling of a diffusive lattice gas

$$
\begin{aligned}
& -\partial_{x} \frac{\rho}{4}=p \frac{\rho}{4}\left(1-\frac{\rho}{4}\right)\left(f_{2}^{\prime}+f_{4}^{\prime}-f_{1}^{\prime}-f_{3}^{\prime}\right)-q\left(f_{3}^{\prime}-f_{1}^{\prime}\right) \frac{\rho^{2}}{16} . \\
& \epsilon^{2}: \\
& \partial_{t} \frac{\rho}{4}+\partial_{x}^{2} \frac{\rho}{8}+\partial_{x} f_{1}^{\prime}=p\left(1-\frac{\rho}{2}\right)\left(f_{2}^{\prime} f_{4}^{\prime}-f_{1}^{\prime} f_{3}^{\prime}\right)+q \frac{\rho}{4}\left(f_{2}^{\prime}+f_{4}^{\prime}\right)\left(f_{3}^{\prime}-f_{1}^{\prime}\right), \\
& \partial_{t} \frac{\rho}{4}+\partial_{x}^{2} \frac{\rho}{8}-\partial_{x} f_{3}^{\prime}=p\left(1-\frac{\rho}{2}\right)\left(f_{2}^{\prime} f_{4}^{\prime}-f_{1}^{\prime} f_{3}^{\prime}\right)-q \frac{\rho}{4}\left(f_{2}^{\prime}+f_{4}^{\prime}\right)\left(f_{3}^{\prime}-f_{1}^{\prime}\right) .
\end{aligned}
$$

Using (A.14) and (A.15) with (A.11), we show that

$$
f_{1}^{\prime}=-f_{3}^{\prime}=-\frac{2}{q \rho^{2}} \partial_{x} \rho=\partial_{x}\left(\frac{2}{q \rho}\right) .
$$

With the same procedure,

$$
f_{2}^{\prime}=-f_{4}^{\prime}=-\frac{2}{q \rho^{2}} \partial_{y} \rho=\partial_{y}\left(\frac{2}{q \rho}\right) .
$$

Substituting (A.18) in (A.16), we obtain

$$
\partial_{t} \frac{\rho}{4}+\partial_{x}^{2} \frac{\rho}{8}+\partial_{x} f_{1}^{\prime}=p\left(1-\frac{\rho}{2}\right)\left(-f_{2}^{\prime 2}+f_{1}^{\prime 2}\right)
$$

and, similarly,

$$
\partial_{t} \frac{\rho}{4}+\partial_{y}^{2} \frac{\rho}{8}+\partial_{y} f_{2}^{\prime}=-p\left(1-\frac{\rho}{2}\right)\left(-f_{2}^{\prime 2}+f_{1}^{\prime 2}\right) .
$$

Summing the two last equation and replacing $f_{1}^{\prime}$ and $f_{2}^{\prime}$ by their expressions in term of density, one obtains the evolution equation for the average density

$$
\partial_{t} \rho+\Delta\left(\frac{\rho}{4}+\frac{4}{q \rho}\right)=0 .
$$

The stationary solution of the density field can be obtained by solving the Laplace equation for $\rho / 4+4 / q \rho$. We compare this solution to the results of the simulation with $\rho_{L}=\rho_{B}=2.4, \rho_{R}=\rho_{T}=1.6$ and deterministic collisions $p=q=1$ (see figure A1).

We naturally observe that the errors are more important near the corners where density of boundary reservoir is discontinuous. The equation (A.22) corresponds to a non-linear diffusion equation

$$
\partial_{t} \rho=\nabla(D(\rho) \nabla \rho),
$$

with diffusivity

$$
\left.\left.\left.\left.D(\rho)=-\frac{1}{4}+\frac{4}{q \rho^{2}}, \quad \geq 0 \forall \rho \in\right] 0: 4\right], q \in\right] 0: 1\right]
$$

The diffusivity has two contributions. The factor $-1 / 4$ is a spurious (anti-diffusive) term called propagation diffusivity [27] which emerges from the discrete nature of the velocity set. The term $\frac{4}{q \rho^{2}}$ will be dominant for $q\left(\frac{\rho}{4}\right)^{2} \ll 1$ and is due to the three particles collisions. The hydrodynamic current of particles is then

$$
j_{\alpha}=-D(\rho) \partial_{\alpha} \rho
$$

The last equation has been checked numerically (see figure A2). The points that don't fit well with the theoretical relation correspond to the nodes near the boundaries. 

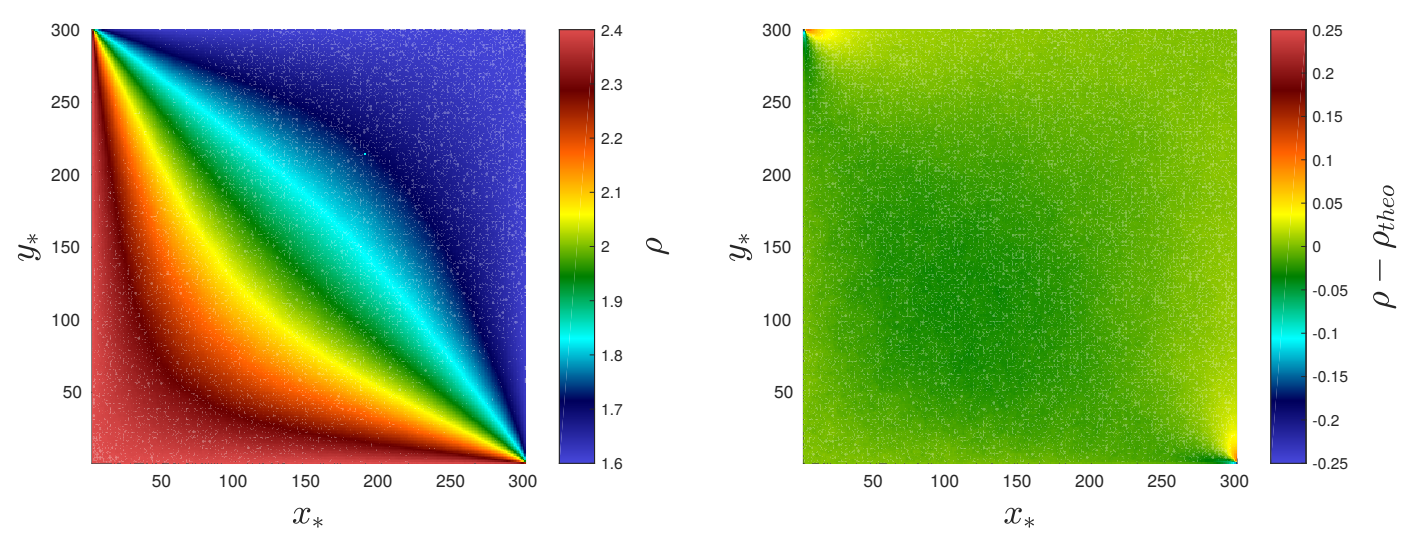

Figure A1. Comparison between the stationary density field solution obtained by a Laplace solver $\rho_{\text {theo }}$ and the average density field from simulation $\rho$. Left: the average density computed over $10^{6}$ time steps. Right: difference with the solution obtained by solving the Laplace equation $\rho_{\text {theo }}$.
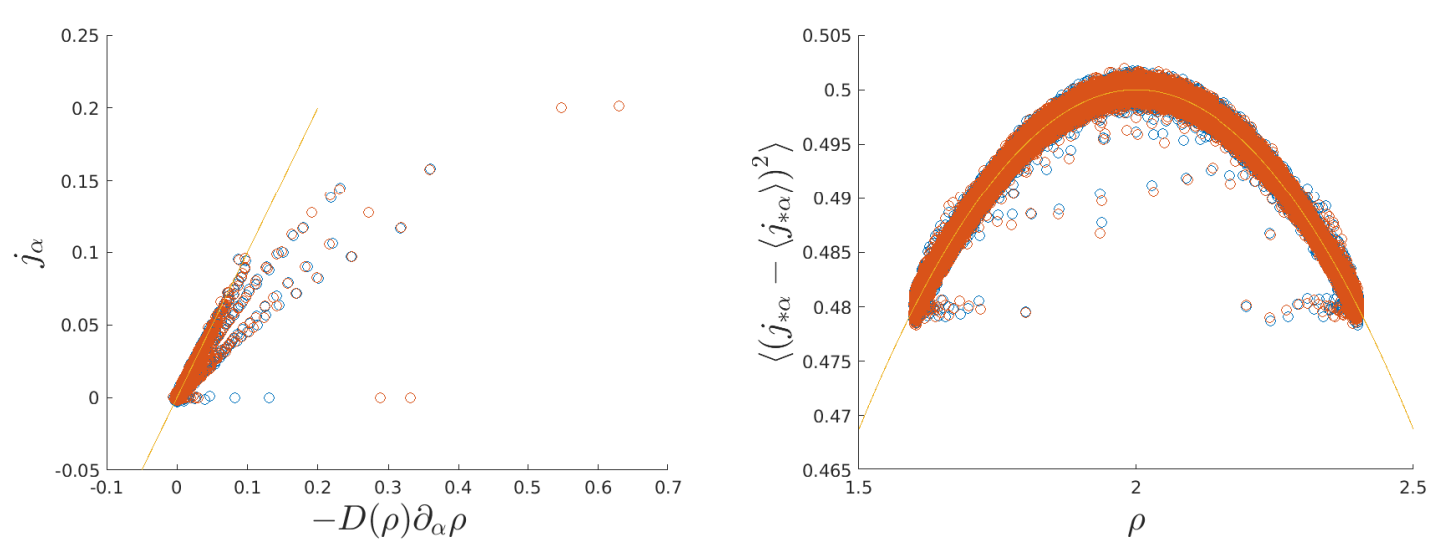

Figure A2. Left: Relation between the average current $j_{\alpha}$ and its theoretical prediction (A.25): $-D(\rho) \partial_{\alpha} \rho$. The line $j_{\alpha}=-D(\rho) \partial_{\alpha} \rho$ is shown in yellow. Right: Relation between the variance of the microscopic current and local average density. The theoretical relation (A.30): $\sigma_{*}^{2}(\rho)=\frac{\rho}{2}\left(1-\frac{\rho}{4}\right)$ is shown in yellow. Blue circles represent $\alpha=x$ and orange circles represent $\alpha=y$.

\section{Appendix A.2. Microscopic fluctuations of the current}

Using the Boltzmann approximation (A.8), it is easy to write the probability to observe a local state $s=\left(s_{i}, i=1, \ldots, 4\right)$ at node $r_{*}$ and time $t_{*}$ using the definition of the mean occupation of channels $N_{i}\left(r_{*}, t_{*}\right)$ :

$$
\mathcal{P}(s)=\prod_{i=1}^{4} N_{i}^{s_{i}}\left(1-N_{i}\right)^{\left(1-s_{i}\right)} .
$$

We can then write the fluctuations of the two components of the current

$$
\left\langle\left(j_{* x}-\left\langle j_{* x}\right\rangle\right)^{2}\right\rangle=N_{1}\left(1-N_{3}\right)+\left(1-N_{1}\right) N_{3}-\left(N_{1}-N_{3}\right)^{2},
$$


Sub-grid modelling of a diffusive lattice gas

$$
\left\langle\left(j_{* y}-\left\langle j_{* y}\right\rangle\right)^{2}\right\rangle=N_{2}\left(1-N_{4}\right)+\left(1-N_{2}\right) N_{4}-\left(N_{2}-N_{4}\right)^{2} .
$$

Using the small perturbation hypothesis (A.11) and keeping only the zero'th order in $\epsilon$, we obtain

$$
\lim _{\epsilon \rightarrow 0}\left\langle\left(j_{* x}-\left\langle j_{* x}\right\rangle\right)^{2}\right\rangle=\lim _{\epsilon \rightarrow 0}\left\langle\left(j_{* y}-\left\langle j_{* y}\right\rangle\right)^{2}\right\rangle \equiv \sigma_{*}^{2}(\rho)=\frac{\rho}{2}\left(1-\frac{\rho}{4}\right) .
$$

We point out that the fluctuation function $\sigma$ is the same as the one computed in the Simple Symmetric Exclusion Process (SSEP) (see [14]) and is typical for models with exclusion. Numerically, the current verifies the relation (A.30) well (see figure A2). As for the mean current, the points that don't fit well with the theoretical relation correspond to the boundary nodes.

\section{Appendix A.3. Violation of the Boltzmann hypothesis}

In practice, the particles can be correlated before collision. To quantify these correlations, we look at the local observables

$$
\begin{aligned}
& n_{p}=n_{2} n_{4}\left(1-n_{1}\right)\left(1-n_{3}\right)-n_{1} n_{3}\left(1-n_{2}\right)\left(1-n_{4}\right), \\
& n_{q_{x}}=-\left(n_{3}-n_{1}\right) n_{2} n_{4}, \\
& n_{q_{y}}=-\left(n_{4}-n_{2}\right) n_{1} n_{3}, \\
& n_{x y}=\left(n_{1}-n_{3}\right)\left(n_{2}-n_{4}\right) .
\end{aligned}
$$

$n_{p}\left(n_{q_{x}}\right.$ or $\left.n_{q_{y}}\right)$ represent the effect of two particles collision (three particles collision along $x$ or $y) . n_{x y}$ is related to the correlation between the horizontal et vertical currents. We note $N_{p}\left(N_{q_{x}}, N_{q_{y}}\right.$ or $\left.N_{x y}\right)$ the value of $\left\langle n_{p}\right\rangle\left(\left\langle n_{q_{x}}\right\rangle,\left\langle n_{q_{y}}\right\rangle\right.$ or $\left.\left\langle n_{x y}\right\rangle\right)$ evaluated in the lattice Boltzmann approximation. So, we evaluate the violation of the Boltzmann approximation by computing the coefficients

$$
\begin{aligned}
& r e_{p}=\frac{\left\langle n_{p}\right\rangle-N_{p}}{N_{p}}, \\
& r e_{q_{x}}=\frac{\left\langle n_{q_{x}}\right\rangle-N_{q_{x}}}{N_{q_{x}}}, \\
& r e_{q_{y}}=\frac{\left\langle n_{q_{y}}\right\rangle-N_{q_{y}}}{N_{q_{y}}}, \\
& r e_{x y}=\frac{\left\langle n_{x y}\right\rangle-N_{x y}}{N_{x y}},
\end{aligned}
$$

which are the relative errors (compared to the Lattice Boltzmann Approximation) of $n_{p}$ $\left(n_{q_{x}}, n_{q_{y}}\right.$ and $\left.j_{* x} j_{* y}\right)$. These coefficients are evaluated at each node (see figure A3). For $r e_{p}$ and $r e_{q_{x}}$ the fluctuations are important. The statistics of $r e_{p}$ are homogeneous inside the system, except near the boundaries where there is a discontinuity of density of the reservoirs. It is globally positive. However, the statistics of $r e_{q_{x}}$ are not homogeneous and we observe important fluctuations near the top and bottom boundaries. These correlations between particles are not significantly modified when we change the size of the lattice. A similar behaviour is observed for $r e_{q_{y}}$. 

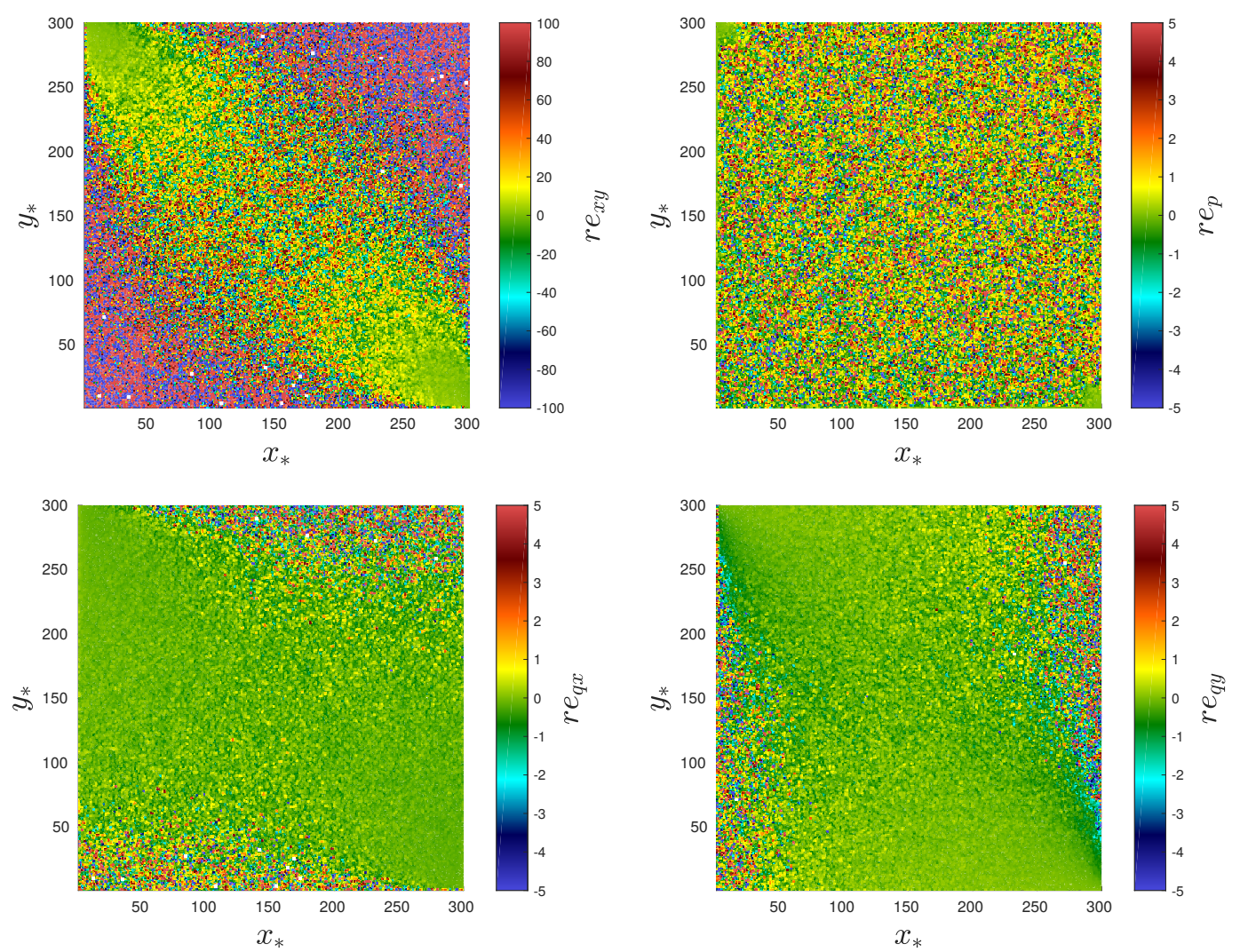

Figure A3. Relative error coefficients defined in (A.35-A.38) that quantify the departure from the Lattice Boltzmann Approximation for our simulation. The fields presented here are smoothed by a Gaussian filter.

\section{References}

[1] Stephen B Pope. Turbulent flows. Measurement Science and Technology, 12(11):2020-2021, oct 2001.

[2] M Lesieur and O Metais. New trends in large-eddy simulations of turbulence. Annual Review of Fluid Mechanics, 28(1):45-82, 1996.

[3] Pierre-Henri Chavanis. A parametrization of two-dimensional turbulence based on a maximum entropy production principle with a local conservation of energy. Fluid Dynamics Research, 46(6):061409, oct 2014.

[4] J. Smagorinsky. General circulation experiments with the primitive equations. Monthly Weather Review, 91(3):99-164, 1963.

[5] U. Frisch, D. d'Humières, B. Hasslacher, P. Lallemand, Y. Pomeau, and J. P. Rivet. Lattice gas hydrodynamics in two and three dimensions. Complex Syst., 1:649-707, 1987.

[6] P Grosfils, JP Boon, and P Lallemand. Spontaneous fluctuation correlations in thermal lattice-gas automata. Physical review letters, 68(7):10771080, February 1992.

[7] Dieter A Wolf-Gladrow. Lattice-gas cellular automata and lattice boltzmann models - an introduction. Lattice-Gas Cellular Automata and Lattice Boltzmann Models, 1725, 012000.

[8] J.-P. Rivet and J. P. Boon. Lattice Gas Hydrodynamics. September 2005.

[9] Kirone Mallick. The exclusion process: A paradigm for non-equilibrium behaviour. Physica A: Statistical Mechanics and its Applications, 418:17 - 48, 2015. Proceedings of the 13th International Summer School on Fundamental Problems in Statistical Physics. 
[10] B. Derrida, E. Domany, and D. Mukamel. An exact solution of a one-dimensional asymmetric exclusion model with open boundaries. Journal of Statistical Physics, 69(3):667-687, Nov 1992.

[11] Alexandre Lazarescu. The physicist's companion to current fluctuations: one-dimensional bulkdriven lattice gases. Journal of Physics A: Mathematical and Theoretical, 48(50):503001, nov 2015.

[12] L. Bertini, A. De Sole, D. Gabrielli, G. Jona-Lasinio, and C. Landim. Macroscopic fluctuation theory for stationary non-equilibrium states. Journal of Statistical Physics, 107(3):635-675, May 2002.

[13] Bernard Derrida. Non-equilibrium steady states: fluctuations and large deviations of the density and of the current. Journal of Statistical Mechanics: Theory and Experiment, 2007(07):P07023P07023, jul 2007.

[14] Bernard Derrida. Microscopic versus macroscopic approaches to non-equilibrium systems. Journal of Statistical Mechanics: Theory and Experiment, 2011(01):P01030, jan 2011.

[15] Lorenzo Bertini, Alberto De Sole, Davide Gabrielli, Giovanni Jona-Lasinio, and Claudio Landim. Macroscopic fluctuation theory. Reviews of Modern Physics, 87, 042014.

[16] Qisu Zou, Shuling Hou, and Gary D. Doolen. Analytical solutions of the lattice boltzmann bgk model. Journal of Statistical Physics, 81(1):319-334, Oct 1995.

[17] J Hardy, Yves Pomeau, and O de Pazzis. Time evolution of a two-dimensional classical lattice system. Physical Review Letters - PHYS REV LETT, 31:276-279, 071973.

[18] J Hardy, O de Pazzis, and Yves Pomeau. Molecular dynamics of a classical lattice gas: Transport properties and time correlation functions. Phys. Rev. A, 13, 051976.

[19] C. Beck and E.G.D. Cohen. Superstatistics. Physica A: Statistical Mechanics and its Applications, $322: 267-275,2003$.

[20] Massimo Germano, Ugo Piomelli, Parviz Moin, and William H. Cabot. A dynamic subgridscale eddy viscosity model. Physics of Fluids A: Fluid Dynamics, 3(7):1760-1765, 1991.

[21] C. Gardiner. Stochastic Methods: A Handbook for the Natural and Social Sciences. Springer Series in Synergetics. Springer Berlin Heidelberg, 2009.

[22] Hugo Touchette. Introduction to dynamical large deviations of markov processes. Physica A: Statistical Mechanics and its Applications, 504:5 - 19, 2018. Lecture Notes of the 14th International Summer School on Fundamental Problems in Statistical Physics.

[23] Jean Pierre Boon, Eric Vanden Eijnden, and David Hanon. A lattice gas automaton approach to 'turbulent diffusion'. Chaos, Solitons \& Fractals, 11(1):187 - 192, 2000.

[24] M R Evans and T Hanney. Nonequilibrium statistical mechanics of the zero-range process and related models. Journal of Physics A: Mathematical and General, 38(19):R195R240, Apr 2005.

[25] C. Beck. Superstatistics: theory and applications. Continuum Mechanics and Thermodynamics, 16(3):293-304, Mar 2004.

[26] Christian Beck. Generalized statistical mechanics for superstatistical systems. Philosophical Transactions of the Royal Society A: Mathematical, Physical and Engineering Sciences, 369(1935):453-465, 2011.

[27] Michel Hénon. Viscosity of a lattice gas. Complex Systems, 1, 1987. 